\section{Hildegard von Bingens Arzneien gegen Frauenleiden aus pharmakologischer Sicht}

\author{
D. Ritzmann, I. Ritzmann
}

Wie wurden Sexualität, Menstruation, Schwangerschaft und Geburt im Hochmittelalter beschrieben? Welches Verständnis von Frauenkrankheiten bestand damals? Was wurde empfohlen und wovor wurde gewarnt? Hildegard von Bingen, Äbtissin aus dem Rheinland, gibt auf diese Fragen bemerkenswerte und oft überraschende Antworten. Die Pflanzen, die sie in ihren naturkundlich-medizinischen Werken [1] zur Therapie von Frauenleiden empfahl, sind in der sogenannten "Volksmedizin" teilweise bis heute in Anwendung. Was wissen wir über diese Pflanzen? Handelt es sich überhaupt noch um dieselben, die Hildegard vor über 800 Jahren beschrieb? Was ist zum Beispiel eine "Rifelbere»? Und gibt es eine wissenschaftliche Begründung für ihre Anwendung?

Und um ein konkretes Beispiel zu nennen: Was empfiehlt Hildegard einer «mulier, quae iniusto multa menstrua inordinate patitur", also einer Frau, die an unzeitigem, starkem Monatsfluss leidet? Diese Frau solle kalte Umschläge anwenden, um innerlich abzukühlen, aber auch Sellerie kochen und sich diese warm auf die Schenkel und den Nabel auflegen. Dadurch könne der nachteilige Blutfluss verringert werden. Auch der häufige Genuss von Wein, einem als "warm" geltenden Getränk, mit eingelegter, ebenfalls wärmespendender Betonie wirke der unzweckmässigen Erwärmung des Blutes entgegen. Weiter empfiehlt Hildegard Massagen und warnt vor zu strenger körperlicher Arbeit.

Es mag erstaunen, dass die Äbtissin - im Gegensatz zu anderen religiösen Autoren - niemals christliche Verfehlungen bzw. göttliche Strafen und nur äusserst selten Geister und Zauberei als Krankheitsursache angibt. Hauptsächlich nennt sie physikalische Fehler in der Mechanik der Körpersäfte, so z.B. ein Zuviel oder Zuwenig, Verstopfungen und Verlegungen sowie Probleme im Wärme- und Kältehaushalt der Humores. Ebenfalls eine wichtige Rolle als krankheitsauslösende Faktoren schreibt sie seelischen Beschwerden zu.

Korrespondenz:

Dr. med. Dorin Ritzmann

Schweighofstrasse 60

CH-8045 Zürich

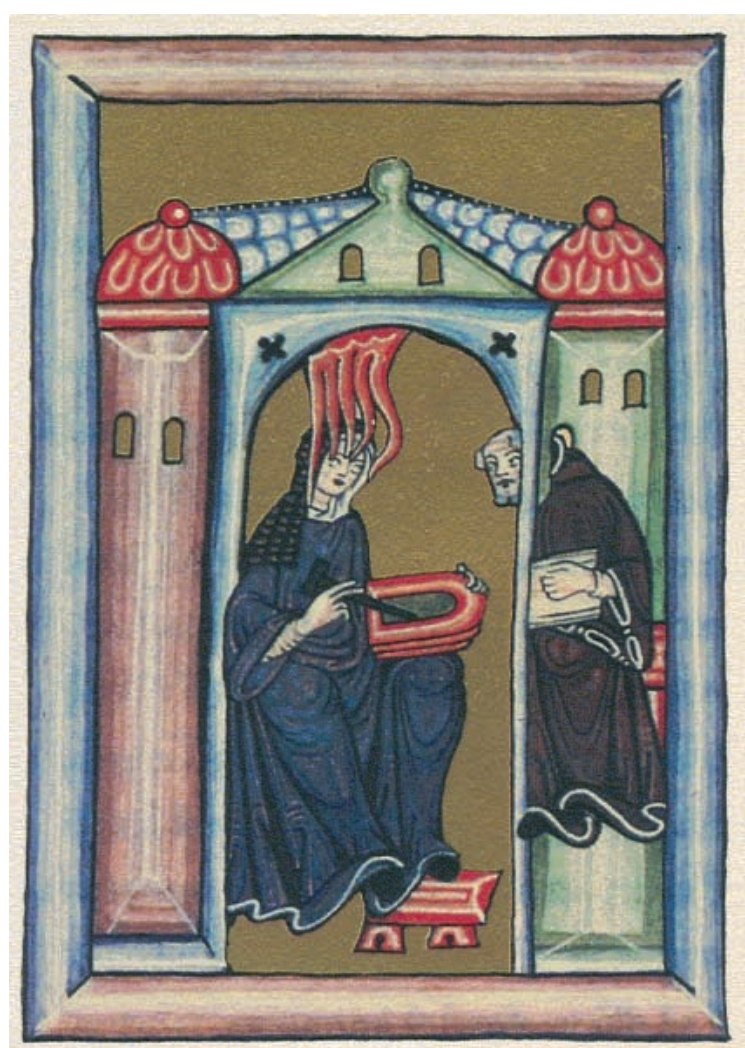

Diese Miniatur aus Hildegardis Scivias (Verlag Brepols I. G. P., Turnhout/B) weist folgende Legende auf: «Hildegardis igneo lumine inflammat. Testimonio Volmari monchi, manus ad scribendum apponit.» Das Liber Scivias entstand in den Jahren 1141-1151 (abgedruckt in: Scherer W. Hildegard von Bingen, Musik und Minnemystik. Freiburg i.B.: Kore-Verlag 1987.)

Dieser Abwendung von einer rein theurgischen, also von Gott bestimmten Krankheitsentstehung entsprechend rät Hildegard nicht einfach zum heilbringenden Gebet. Sie macht profane therapeutische Vorschläge, so die Anwendung von Pflanzen, die mit ihren Eigenschaften wie Wärme oder Kälte wieder Ordnung ins gestörte System des menschlichen Körpers bringen sollen. Damit weist Hildegards Medizin Vorstufen eines Denkmodells auf, das auf "Naturwissen" basiert und aus dem sich ein naturwissenschaftliches Vorgehen entwickeln konnte, ein für das Hochmittelalter erstaunlicher Befund!

So beeindruckend Hildegards Werk, so vorsichtig und kritisch sollte der Umgang mit der Umsetzung ihrer Empfehlungen sein. Fünfundzwanzig Pflanzen empfiehlt sie bei frauenheilkundlichen Erkrankungen. Von diesen fünfundzwanzig Pflanzen sind sieben in der von Hildegard beschriebenen Indikation nach modernem Wissen effektiv wirksam. Weitere zwölf Pflanzen weisen zumindest Inhaltsstoffe auf, die der von Hildegard angegebenen Wirkung entsprechen, allerdings ohne dass ein direkter Wirkungsnachweis vorliegt. Von den übrigen sechs Pflanzen sind zwei nicht sicher bestimmbar, und vier wurden bisher nicht näher untersucht, so dass über ihre Wirkung noch nichts Verlässliches gesagt werden kann. 
Damit lässt sich zwar belegen, dass Hildegard immerhin mindestens neunzehn von fünfundzwanzig Pflanzen nach heutigen rein pharmakologischen Kenntnissen korrekt indiziert hatte. Dieser Befund sagt allerdings nichts über die medizinisch-therapeutische Anwendung dieser Drogen aus: Die sieben eindeutig wirksamen Arzneipflanzen werden heute nicht mehr angewandt, weil ihre Anwendung zu allergisierenden oder sogar toxischen Nebenwirkungen führen kann. [2]

\section{Literatur}

1 Beigezogene Werke Hildegard von Bingens in Lateinisch und deutscher Übersetzung: Schipperges H (Hrsg.). Berliner Fragmente (1956; übers. M.-L. Portmann, 1987); Daremberg C, Reuss FA (Hrsg.). Physica (1855; übers. M.-L. Portmann, 1991); Kaiser P. Causae et Curae (1905; übers. H. Schulz, 1933).

2 Genaueres hierzu in: Ritzmann Schilt LD. Hildegard von Bingen: Pflanzliche Heilmittel mit gynäkologischgeburtshilflicher Indikation. Dietikon; 1994

(Zürcher Medizingeschichtliche Abhandlungen 259). 